\section{Tarsal-tunnel Syndrome, a Presenting Feature of Rheumatoid Arthritis}

British Medical fournal, 1970, 3, 32

The tarsal-tunnel syndrome, produced by compression of the posterior tibial nerve beneath the flexor retinaculum below the medial malleolus of the ankle, has been described by Kopell and Thompson (1960), Keck (1962), Lam (1962), Kojima (1963), McGill (1964), Babbage (1965), and Paterson (1966). We can find no previous reference to this condition occurring in association with rheumatoid arthritis or of being the presenting feature of the disease, as a carpal-tunnel syndrome may sometimes be.

\section{Case Report}

A 51-year-old woman who worked full time standing all day as a checker clerk presented with pain, numbness, and tingling in both feet which had come on insidiously some six months previously and was particularly severe at night, frequently waking her.

General examination showed nothing worth remark, and in particular all peripheral joints were normal. there was diminution of sensation to pin-prick in the medial aspect of both feet and extending into the first three toes, with maximum density in the great toes. Muscle weakness or wasting was not evident, but there was tenderness over the surface marking of both posterior tibial nerves just below the medial malleoli, tapping over the nerves did not aggravate paraesthesiae. There was no swelling in the area, the long flexor and tibialis posterior tendons were working normally; there were no foot deformities, and $x$-ray pictures of the area were normal.

The distribution of sensory loss and the localized nerve tenderness indicated a diagnosis of compression of the posterior tibial nerves in the tarsal tunnels. Motor and sensory nerve conduction velocities on the medial and lateral popliteal nerves of the right leg proved to be normal, but in the light of similar normal findings in undoubted cases of median nerve compression in the carpal tunnel we thought that these findings did not negative the diagnosis. Accordingly, $1.5 \mathrm{ml}$. of prednisolone tertiary butylacetate was injected into the left tarsal tunnel. Three weeks later, the patient reported dramatic improvement in her symptoms within 48 hours of the injection, and this improvement had been maintained. She had no pain and complained only of very mild paraesthesiae. The right tarsal tunnel was similarly treated, the response being identical. When seen a month later she had only mild paraesthesiae in both feet; the injection procedure was repeated on both sides at one sitting. Three weeks later she was completely symptomless; the only abnormal sign was a small area of hypoaesthesia to pin-prick over both great toes. It was at this stage that we wondered whether this episode might be the presenting feature of rheumatoid arthritis even though the patient had no symptoms or signs in her peripheral joints, though three years previously she had had pain and stiffness of the left shoulder lasting about six months and then completely subsiding. The E.S.R. proved normal but the differential agglutination titre (D.A.T.) was $1: 512$. The hands were radiographed but showed no abnormality. A month later her D.A.T. had risen to $1: 2,048$, the E.S.R. being still normal.

Six months after her original presentation and a year after the onset of the first symptoms in the feet, she had developed a small nodule in an extensor tendon over the right carpus, the E.S.R. was slightly raised, and D.A.T. titre was $1: 2,048$. Two months later she complained of morning stiffness and had developed mild pain and swelling of all her proximal interphalangeal joints; the E.S.R. was still slightly raised, and the D.A.T. titre remained at $1: 2,048$. Ibuprofen $600 \mathrm{mg}$. daily gave good relief from joint symptoms, her rheumatoid disease has remained minimal, and she has had no recurrence of paraesthesiae in the feet during 18 months' observation.

\section{COMment}

The largest series of tarsal-tunnel syndromes has been reported by Lam (1967). None of his 10 cases had clinical rheumatoid arthritis, but he noted swelling over four tarsal tunnels, in three cases the condition was bilateral, and three also had a carpal-tunnel syndrome. The most likely explanation of compression of the posterior tibial nerve beneath the flexor retinaculum in rheumatoid arthritis is that rheumatoid tenovaginitis affecting the sheaths of the tibialis posterior or the long flexor tendons causes swelling and hence compression; in our case, however, there was no clinical evidence of this. Valgus deformity of the foot secondary to arthritis of the tarsal joints and weakness of the short muscles of the foot is common in rheumatoid arthritis and could be responsible for increased traction on the posterior tibial nerve turning a corner below the malleolus, but again there was no evidence of this in our case. Lastly, inflammation and swelling of the fibrous retinaculum itself is a possibility. The dramatic relief following local corticosteroid certainly suggests that inflammatory swelling of some sort was the cause of the compression.

All the cases described in the literature quoted have been treated by surgical decompression, usually with satisfactory results, but corticosteroid injection beneath the retinaculum is such a simple and quick procedure that we suggest that it should constitute the first line of treatment.

It is possible that this condition occurs not infrequently in rheumatoid arthritis and that its detection is masked by the many other causes of pain in the foot in this disease. Therefore, its presence may be detected only by awareness of the phenomenon and by asking leading questions about the presence of paraesthesiae on the medial side of the foot and searching for a sensory deficit. We are now carrying out a survey to determine the incidence of this and other entrapment neuropathies in rheumatoid arthritis.

Our thanks are due to Dr. John Graham, of the department of neurology, for carrying out the nerve conduction studies.

\section{KENNETH LLOYD, F.R.C.P. AMrit Agarwal, M.R.C.P. \\ Department of Physical Medicine and Rheumatology,} United Cardiff Hospitals.

\section{REFERENCES}

Babbage, N. F. (1965). Medical fournal of Australia, 1, 764 Keck, C. (1962). Fournal of Bone and foint Surgery, 44A, 180 Kojima, T. (1963). Tohoku fournal of Orthopaedics and Traumatology, $7 / 2,214$.

Kopell, H. P., and Thompson, W. A. L. (1960). New England fournal of Medicine, 262, 56.

Lam, S. J. S. (1962). Lancet, 2, 1354

Lam. S. I. S. (1967). Foumal of Bone and Foint Surgery, 49B, 87.

McGill, D. A. (1964). Proceedings of the Royal Society of Medicine, 57, 1125

Patersnn, D. C. (1966). Medical fournal of Australia, 2, 421. 